\title{
A GROUP WITH VERY STRANGE DECOMPOSITION PROPERTIES
}

\author{
M. J. DUNWOODY and J. M. JONES
}

Dedicated to M. F. (Mike) Newman on the occasion of his 65 th birthday

(Received 11 September 1998; revised 21 April 1999)

Communicated by E. A. O'Brien

\begin{abstract}
An example is given of a finitely generated group $G$ for which $G \cong G *_{C} G$, where $C$ is infinite cyclic. 1991 Mathematics subject classification (Amer. Math. Soc.): primary 20E08; secondary 20E06, $20 \mathrm{~F} 05$.
\end{abstract}

\section{Introduction}

We give an example of a finitely generated group $G$ for which $G \cong G *_{C} G$, where $C$ is infinite cyclic and $C \neq G$.

This example is probably 'optimal' for bad behaviour of amalgamated products. In a previous paper [2] we have given an example of a group $G$ for which $G \cong$ $A *_{C} G, A \neq C$. The reader is referred to [2] for a discussion of such examples and the folding sequence technique used in their construction. As we remarked in [2] we had tried unsuccessfully to produce such an example with $A \cong G$. We can now present such an example.

A similar construction was used in [1] to produce 2-generator inaccessible groups. Factoring out by a central cyclic subgroup of our new example will also produce an inaccessible group.

Although it is not possible to find a finitely generated group $G$ for which $G \cong G * G$, $G \neq 1$, the second author [3] gave an example of a finitely generated group $G$ for which $G \cong G \times G, G \neq 1$.

(C) 1999 Australian Mathematical Society 0263-61 15/99 $\$$ A2.00+0.00 


\section{The construction}

As in [2] our example is constructed using an infinite folding sequence. The construction depends on the existence of a lattice of groups with certain properties. Thus we show that there is a lattice of groups as in Figure 1, and also satisfying the following three properties:

(i) $H \cong \mathbb{Z}$

(ii) $A_{1}$ and $B_{1}$ are generated by their intersections with $A$ and $B$

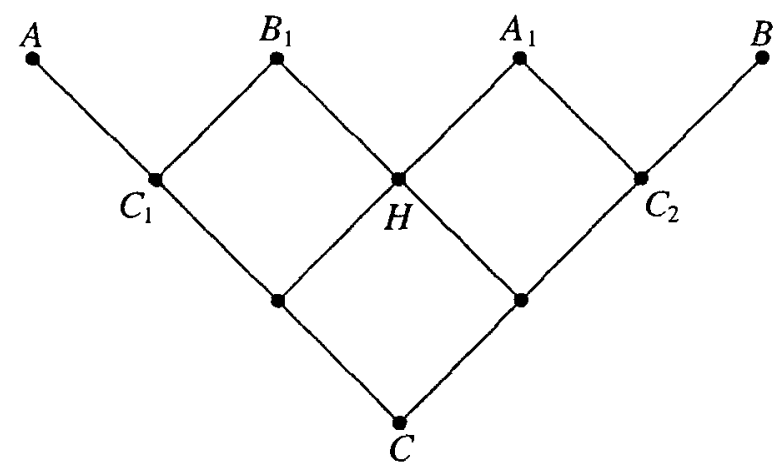

FIGURE 1.

(iii) The amalgams in Figure 2 are isomorphic.
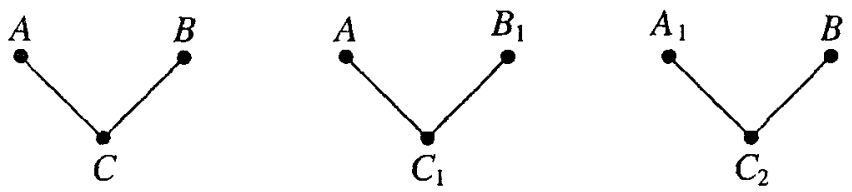

FIGURE 2.

We note from (iii) that $A$ and $A_{1}$ are isomorphic, as are $B$ and $B_{1}$. First we note some other consequences of conditions (i), (ii) and (iii) for the groups $A$ and $A_{1}$.

By (i), $H$ is infinite cyclic, and hence so is every subgroup of $H$. In particular, $C$ is infinite cyclic, say $C=\langle c\rangle$. Using (iii), we see that $C_{1}$ and $C_{2}$ are isomorphic to $C$, so we may set $C_{1}=\langle v\rangle$ and $C_{2}=\langle a\rangle$. Next we use (ii) to deduce that $A_{1}=\left\langle H, C_{2}\right\rangle$, so that if we set $H=\langle h\rangle$, we may conclude that $A_{1}=\langle h, a\rangle$, and in a similar way $B_{1}=\langle h, v\rangle$.

Our aim will be to build 'templates' from which we can construct $A, A_{1}, B$ and $B_{1}$, and then we will fit these groups together to form a diagram as in Figure 1 . Let $m, n, s, t$ be integers greater than three with $m$ and $s$ coprime, and let $k=m t$ and 
$l=n s$. We also demand that $n<k$ and that $t<l$. To facilitate later calculations, we will use the specific values $m=5, n=19, s=7, t=13$.

Let $L$ be the locally cyclic group

$$
\left.L=\left\langle\ldots, v_{-2}, v_{-1}, v_{0}, v_{1}, v_{2}, \ldots\right| v_{i}^{k}=v_{i+1} \text { for all } i \in \mathbb{Z}\right\rangle,
$$

let $M$ be the subgroup of $L$ generated by the set $\left\{v_{i}^{n} \mid i \in \mathbb{Z}\right\}$, and let $M_{1}$ be an isomorphic copy of $M$. As $n<k, M$ is a proper subgroup of $L$. For each integer $i$ let $\left\langle x_{i}\right\rangle$ be a cyclic group of order $m$, and form the free product $X=*_{i \in \mathbb{Z}}\left\langle x_{i}\right\rangle$. The group $P=P_{k, m, n}(\mathbf{v}, \mathbf{x})$ is the amalgamated free product of $L$ with the direct product $X \times M_{1}$, amalgamating the isomorphic groups $M$ and $M_{1}$. Thus the amalgamated subgroup $M=M_{1}$ is central in $P$. We next propose to factor out some relations from $P$, in such a way as to ensure that each pair $v_{i}, x_{i}$ generates the whole group. When extra relations are imposed on a group there is a danger that there will be an unexpected amount of collapse of the original group. To check that our relations have not caused much collapse, we use small cancellation theory over the amalgamated free product $P$. An account of the theory we need can be found in Chapter 5 of [4].

For each $i \in \mathbb{Z}$, we impose the following relations on $P$ :

(i) $x_{i-1}\left(x_{i} v_{i}^{-t}\right)\left(x_{i}^{2} v_{i}^{-2 t}\right)\left(x_{i} v_{i}^{-t}\right)^{2}\left(x_{i}^{2} v_{i}^{-2 t}\right)\left(x_{i} v_{i}^{-t}\right)^{3} \cdots\left(x_{i} v_{i}^{-t}\right)^{80}\left(x_{i}^{2} v_{i}^{-2 t}\right)$,

(ii) $x_{i+1}\left(x_{i} v_{i}^{-t}\right)^{81}\left(x_{i}^{2} v_{i}^{-2 t}\right)\left(x_{i} v_{i}^{-t}\right)^{82}\left(x_{i}^{2} v_{i}^{-2 t}\right)\left(x_{i} v_{i}^{-t}\right)^{83} \cdots\left(x_{i} v_{i}^{-t}\right)^{160}\left(x_{i}^{2} v_{i}^{-2 t}\right)$,

(iii) $v_{i-1}\left(x_{i} v_{i}^{-t}\right)^{161}\left(x_{i}^{2} v_{i}^{-2 t}\right)\left(x_{i} v_{i}^{-t}\right)^{162}\left(x_{i}^{2} v_{i}^{-2 t}\right)\left(x_{i} v_{i}^{-t}\right)^{163} \cdots\left(x_{i} v_{i}^{-t}\right)^{240}\left(x_{i}^{2} v_{i}^{-2 t}\right)$.

We now consider the symmetrised set $R$ generated by these relations. We note that, if $c_{1} c_{2} c_{3} \cdots c_{n}$ is a reduced, cyclically reduced element of an amalgamated free product whose amalgamated subgroup is central, then the cyclically reduced conjugates of $c_{1} c_{2} c_{3} \cdots c_{n}$ can be obtained simply by cyclic permutation. Moreover, if two reduced, cyclically reduced words $c_{1} c_{2} c_{3} \cdots c_{n}$ and $d_{1} d_{2} d_{3} \cdots d_{m}$ share a piece in common then $d_{1}^{-1} c_{1}, d_{2}^{-1} c_{2}, \ldots d_{r}^{-1} c_{r}$ all lie in the amalgamated subgroup, where $r$ is the length of the piece. In our example, using the fact that the factor $X \times M_{1}$ is a direct product, we see that if two elements of $R$ have a piece in common, then the sequence of entries from $X$ appearing in them must agree for the length of the piece. With this in mind, it is not difficult to see that $R$ satisfies the small cancellation condition $C^{\prime}(1 / 10)$. Thus the factors $L$ and $X \times M_{1}$ are embedded in $P /\langle R\rangle^{P}=G_{k, m, n, t}(\mathbf{v}, \mathbf{x})$, say. In particular, each $v_{i}$ has infinite order.

Since relations (i), (ii) and (iii) hold for each $i \in \mathbb{Z}$, it is clear that the map which takes each $v_{i}$ to $v_{i+1}$ and each $x_{i}$ to $x_{i+1}$ is an automorphism of $G_{k, m, n, t}(\mathbf{v}, \mathbf{x})$. Moreover, $G_{k, m, n, t}(\mathbf{v}, \mathbf{x})$ is generated by any pair $v_{i}, x_{i}$ since relations (i) and (iii) show that $\left\langle x_{i}, v_{i}\right\rangle \supseteq\left\langle x_{i-1}, v_{i-1}\right\rangle$, and hence that $\left\langle x_{i}, v_{i}\right\rangle \supseteq\left\langle x_{i-1}, v_{i-1}\right\rangle \supseteq\left\langle x_{i-2}, v_{i-2}\right\rangle \supseteq \cdots$, while relation (ii) together with the relation $v_{i}^{k}=v_{i+1}$ of $L$ shows that $\left\langle x_{i}, v_{i}\right\rangle \supseteq$ $\left\langle x_{i+1}, v_{i+1}\right\rangle$, and hence that $\left\langle x_{i}, v_{i}\right\rangle \supseteq\left\langle x_{i+1}, v_{i+1}\right\rangle \supseteq\left\langle x_{i+2}, v_{i+2}\right\rangle \supseteq \cdots$.

We now define $u_{i+1}=x_{i} v_{i}^{n t}$. Recalling that $v_{i}^{n}$ is central in $G_{k, m, n, t}(\mathbf{v}, \mathbf{x})$, we see that $u_{i+1}^{m}=x_{i}^{m} v_{i}^{n t m}=v_{i}^{n k}=v_{i+1}^{n}$ for each $i \in \mathbb{Z}$ and hence that each $u_{i}$ has infinite order. 
We wish to show that each pair $u_{i+1}, v_{i+1}$ generates $G_{k, m, n, t}(\mathbf{v}, \mathbf{x})$. Since $v_{i+1}$ and $x_{i+1}$ are a pair of generators, relation (ii) shows that it is sufficient to prove that $x_{i} v_{i}^{-t}$ and $x_{i}^{2} v_{i}^{-2 t}$ both lie in $\left\langle u_{i+1}, v_{i+1}\right\rangle$. Now $u_{i+1}=x_{i} v_{i}^{n t}$ and $v_{i+1}=v_{i}^{k}=v_{i}^{m t}$ so $\left\langle u_{i+1}, v_{i+1}\right\rangle \ni$ $u_{i+1} v_{i+1}^{-4}=x_{i} v_{i}^{(n-4 m) t}=x_{i} v_{i}^{-t}$ as $n=19$ and $m=5$. Thus $x_{i} v_{i}^{-t} \in\left\langle u_{i+1}, v_{i+1}\right\rangle$. Similarly, $u_{i+1}^{2}=x_{i}^{2} v_{i}^{2 n t}$ ( since $v_{i}^{n}$ is central), so $u_{i+1}^{2} v_{i+1}^{-8}=x_{i}^{2} v_{i}^{(2 n-8 m) t}=x_{i}^{2} v_{i}^{-2 t}$, so that $\left\langle u_{i+1}, v_{i+1}\right\rangle$ contains both $x_{i} v_{i}^{-t}$ and $x_{i}^{2} v_{i}^{-2 t}$ and hence $x_{i+1}$. Thus we have shown that $u_{i+1}$ and $v_{i+1}$ generate $G_{k, m, n, t}(\mathbf{v}, \mathbf{x})$ as required.

$G_{k, m, n, t}(\mathbf{v}, \mathbf{x})$ is the template for the groups $A$ and $A_{1}$. As a template for $B$ and $B_{1}$ we use $G_{l, s, t, n}(\mathbf{a}, \mathbf{y})$, where, as before, the map sending each $a_{i}$ to $a_{i+1}$ and each $y_{i}$ to $y_{i+1}$ is an automorphism of $G_{l, s, t, n}(\mathbf{a}, \mathbf{y})$, and each pair $\left\{a_{i}, y_{i}\right\}$ generates $G_{l, s, t, n}(\mathbf{a}, \mathbf{y})$. Defining $b_{i+1}=y_{i} a_{i}^{t n}$, so that $b_{i+1}^{s}=a_{i+1}^{t}$, and noting that $b_{i+1} a_{i+1}^{-2}=y_{i} a_{i}^{(t-2 s) n}=$ $y_{i} a_{i}^{-n}$ and $b_{i+1}^{2} a_{i+1}^{-4}=y_{i}^{2} a_{i}^{(2 t-4 s) n}=y_{i}^{2} a_{i}^{-2 n}$ since $t=13$ and $s=7$, we see that $b_{i+1}$ and $a_{i+1}$ generate $G_{l, s, t, n}(\mathbf{a}, \mathbf{y})$.

We can now construct the required diagram. We take a copy $A_{1}$ of $G_{k, m, n, t}(\mathbf{v}, \mathbf{x})$ and a copy $B_{1}$ of $G_{l, s, t, n}(\mathbf{a}, \mathbf{y})$, and form the amalgamated free product $B_{1} *_{b_{0}=u_{0}} A_{1}$. Since $m$ and $s$ are coprime the resulting group, G, has a pattern of subgroups as in the following diagram:

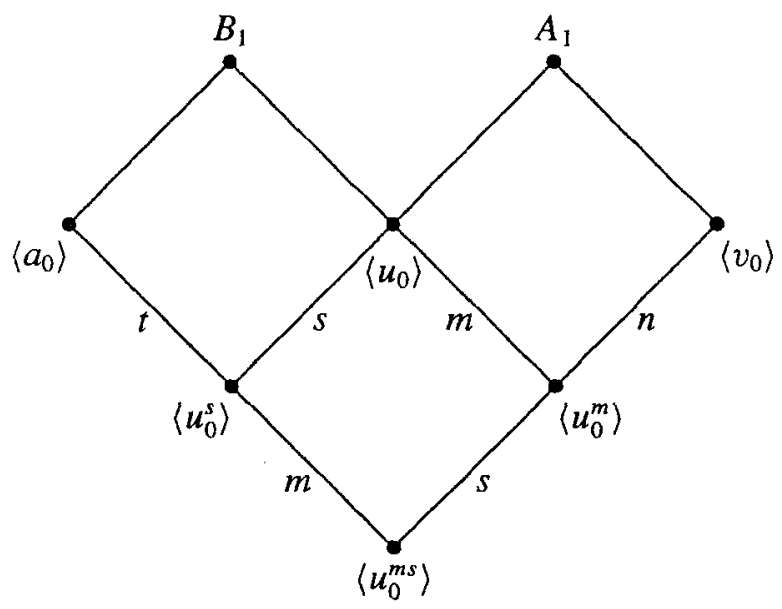

FIGURE 3.

Finally, we take groups $A=G_{k, m, n, t}\left(\mathbf{v}^{\prime}, \mathbf{x}^{\prime}\right)$ and $B=G_{l, s, t, n}\left(\mathbf{a}^{\prime}, \mathbf{y}^{\prime}\right)$, and form the amalgamated free product $A *_{v_{0}^{\prime}=a_{0}} G *_{v_{0}=a_{0}^{\prime}} B$. This group has a pattern of subgroups as in Figure 1, so it only remains to check that the required conditions (i), (ii) and (iii), given for that diagram, hold. The group labelled $H$ in Figure 1 is $\left\langle u_{0}\right\rangle$, so condition (i) certainly holds. For (ii), we need to check that that $A_{1}=\left\langle u_{0}^{s}, v_{0}\right\rangle$ and $B_{1}=\left\langle b_{0}^{m}, a_{0}\right\rangle$. Now $\left\langle u_{0}^{s}, v_{0}\right\rangle$ contains both $u_{0}^{s}$ and $v_{0}^{n}=u_{0}^{m}$ and so contains $u_{0}$ since $m$ and $s$ are coprime. Thus $\left\langle u_{0}^{s}, v_{0}\right\rangle=\left\langle u_{0}, v_{0}\right\rangle=A_{1}$. A similar argument holds for $B_{1}$, and so condition (ii) is satisfied. Now consider condition (iii). Recall 
that there is an automorphism of $A=G_{k, m, n, t}\left(\mathbf{v}^{\prime}, \mathbf{x}^{\prime}\right)$ which takes $u_{0}^{\prime}$ to $u_{1}^{\prime}$ and $v_{0}^{\prime}$ to $v_{1}^{\prime}=v_{0}^{\prime k}$. Composing the map from $A_{1}$ to $A$ which takes $u_{0}$ to $u_{0}^{\prime}$ and $v_{0}$ to $v_{0}^{\prime}$ with this automorphism we have an isomorphism between $A_{1}$ and $A$ which takes $u_{0}$ to $u_{1}^{\prime}$ and $v_{0}$ to $v_{0}^{\prime k}$. Thus it carries the subgroup $C_{2}=\left\langle v_{0}\right\rangle$ of $A_{1}$ to the subgroup $C=\left\langle v_{0}^{\prime k}\right\rangle$ of $A$. The automorphism of $B$ which takes $a_{0}^{\prime}$ to $a_{1}^{\prime}$ and $b_{0}^{\prime}$ to $b_{1}^{\prime}$ also carries $C_{2}$ to $C$, so we can combine the two isomorphisms to show that the first two amalgams of Figure 2 are isomorphic. A very similar argument shows that the first and third amalgams of Figure 2 are isomorphic, and so condition (iii) holds. In the notation of Figure $1, u_{0}$ plays the role of $h, a_{0}=v_{0}^{\prime}$ that of $v$, and $v_{0}=a_{0}^{\prime}$ that of $a$.

Consider the infinite folding sequence shown in Figure 4.

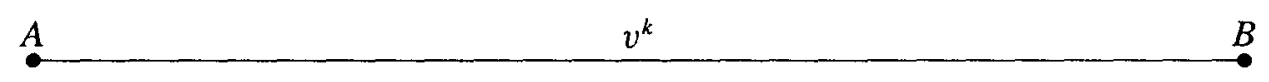

(subdivision)

A

$B$

(Type II folds)

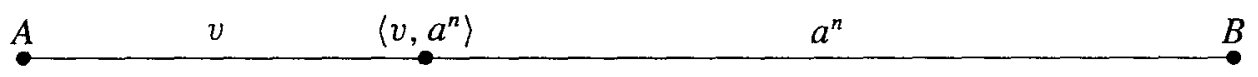

(vertex morphism)

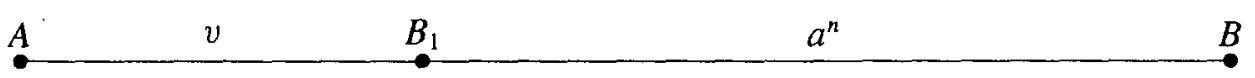

(subdivision and Type II folds)

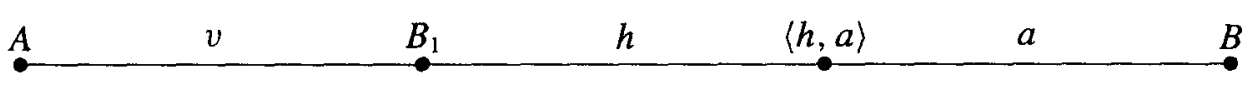

(vertex morphism)

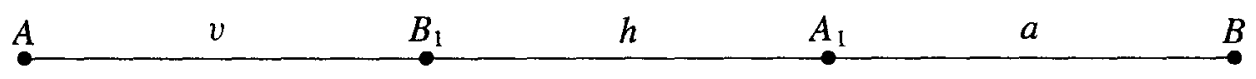

(repeating process infinitely many times on Cantor Set)

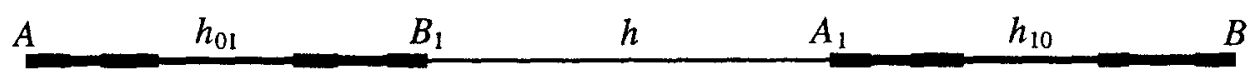

FIGURE 4.

The $n$-th term is a simplicial tree $T_{n}$ acted on by a group $G_{n}$. There are surjective homomorphisms

$$
G_{0} \rightarrow G_{1} \rightarrow G_{2} \rightarrow \cdots
$$


The group $G$ is the direct limit of this sequence.

The group $G$ that we have constructed has a lattice of subgroups as in Figure 5. By considering this lattice it can be seen that the construction of $G$ is like the construction of a jigsaw puzzle. All the jigsaw pieces are similar to the piece given in Figure 1 . To get from $G_{n}$ to $G_{n+1}$ one has to attach $2^{n}$ pieces to the puzzle, all of these pieces are congruent and fit into $2^{n} \mathrm{~V}$-shaped gaps in the upper boundary. The fitting of each piece involves a sequence of folds in the tree $T_{n}$ similar to the folds described in Figure 4.

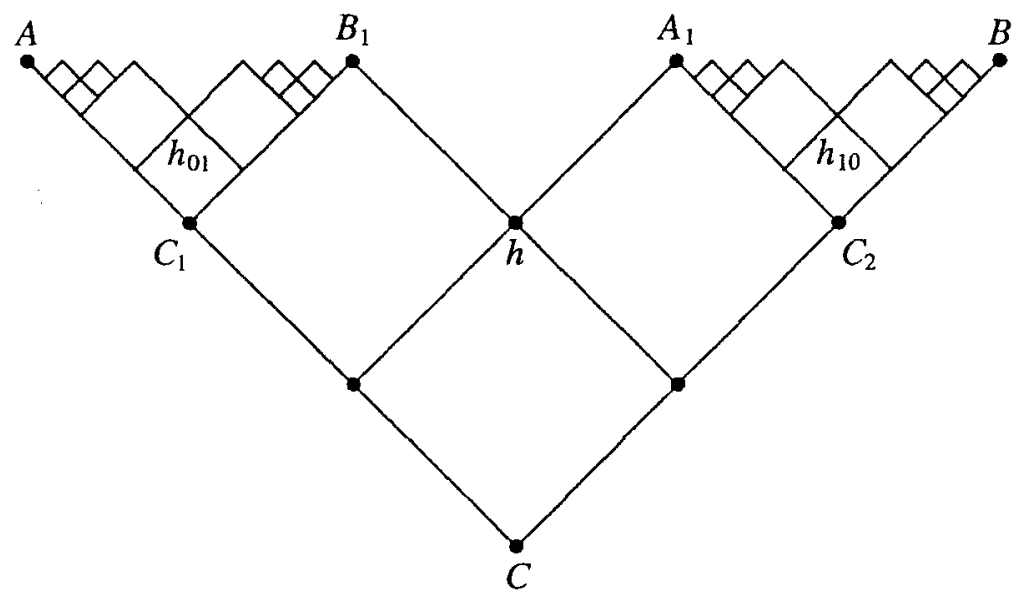

FIGURE 5.

It can be seen that $G \cong G *_{H} G$. Note that the isomorphism of $G$ to the first factor fixes $A$ and carries $B$ to $B_{1}$ and $H=\langle h\rangle$ to $\left\langle h_{01}\right\rangle$. The isomorphism to the second factor fixes $B$ and carries $A$ to $A_{1}$ and $H$ to $\left\langle h_{10}\right\rangle$.

\section{References}

[1] M. J. Dunwoody, 'Inaccessible groups and protrees', J. Pure Appl. Algebra 88 (1993), 63-78.

[2] M. J. Dunwoody and J. M. Jones, 'A group with strange decomposition properties', J. Group Theory 1 (1998), 301-305.

[3] J. M. T. Jones, 'Direct products and the Hopf property', J. Austral. Math. Soc. 17 (1974), 174-196.

[4] R. C. Lyndon and P. E. Schupp, Combinatorial group theory (Springer, Berlin, 1977).

Faculty of Mathematics

University of Southampton

Southampton, SO17 1BJ

UK 